\title{
Multigraf: Aplicativo Facilitador na Aprendizagem da Multiplicação e Interpretação de Gráficos
}

\author{
Regiano Monte Viana ${ }^{1}$, José Ailton Batista da Silva ${ }^{1}$, Tulio Vidal Rolim ${ }^{2}$, Rangel \\ Henrique Félix ${ }^{3}$, Paulo César Henrique da Silva ${ }^{1}$, Arthur Cézar Valentim de Melo ${ }^{1}$, \\ Pedro Luis Saraiva Barbosa ${ }^{1}$
}

\author{
${ }^{1}$ Instituto Federal de Educação, Ciência e Tecnologia do Ceará (IFCE) \\ Cedro - CE, Brasil \\ ${ }^{2}$ Universidade Federal do Ceará (UFC) \\ Fortaleza - CE, Brasil \\ ${ }^{3}$ Instituto Federal de Educação, Ciência e Tecnologia do Ceará (IFCE) \\ Jaguaribe - CE, Brasil \\ \{ailton.ifce, tulio.xcrtf, rangel.crede1,pch452079\}@gmail.com, \\ \{arthurcvm, regiano_viana\}@hotmail.com,pedro.barbosa@ifce.edu.br
}

\begin{abstract}
Many of the activities involving mathematics are seen by many students as complex and discouraging, often making this area of knowledge uninviting to study. However, with the increasing advancement of technology, it is presumed that the educational field will use this powerful resource as a means to aid pedagogical practices. In this sense, the present study aims to identify the contributions in the learning of the multiplication and interpretation of graphs after the use of an educative app titled "Multigraf". The study was carried out with twenty students in a high school located in the city of Icó-Ceará, having as instrument of collection the application of a questionnaire in two different moments. After the collection, it was possible to identify that there were improvements in the learning regarding multiplication and interpretation of the graphs in the participating students after using the app.
\end{abstract}

Resumo. Muitas das atividades que envolvem matemática são vistas por boa parte dos alunos como complexas e desestimulantes, tornando muitas vezes essa área do conhecimento pouco convidativa ao seu estudo. Contudo, com o crescente avanço da tecnologia, é presumível que o campo educacional venha a utilizar este poderoso recurso como meio de auxílio às práticas pedagógicas. Neste intuito, o presente estudo busca visa identificar as contribuições na aprendizagem da multiplicação e interpretação de gráficos após a utilização de um aplicativo educativo intitulado "Multigraf". O estudo foi realizado com vinte alunos em uma escola de ensino fundamental situada no município de Icó-Ceará, tendo como instrumento de coleta a aplicação de um questionário em dois momentos distintos. Após a coleta, foi possível identificar que houveram melhoras na aprendizagem quanto à multiplicação e interpretação dos gráficos nos alunos participantes após utilização do aplicativo. 
VII Congresso Brasileiro de Informática na Educação (CBIE 2018)

Anais dos Workshops do VII Congresso Brasileiro de Informática na Educação (WCBIE 2018)

\section{Introdução}

As atividades escolares que envolvem a matemática são vistas por boa parte dos alunos como importunas e complexas, tornando essa área do conhecimento pouco convidativa ao seu estudo. Atualmente, com o surgimento das ferramentas tecnológicas, observa-se um mundo tecnológico baseado na maioria das vezes em recursos informatizados, recursos estes que possibilitam engrandecer o conhecimento matemático dentro dessa nova metodologia de ensino, que vem conquistando espaço e força com o passar do tempo.

$\mathrm{Na}$ busca pela inovação, criatividade e motivação nas práticas pedagógicas, jogos digitais têm sido considerados um importante recurso tecnológico de apoio à educação, uma vez que aumenta o engajamento e a motivação do aprendiz [Pantaleão, Amaral e Silva 2017].

Os recursos tecnológicos vieram para facilitar o aprendizado em diversas áreas do conhecimento, tal como a matemática, tornando-os passivos na construção de ideias para aproveitamento das práticas de ensino adotadas. Aplicativos com boa usabilidade consegue atrair a atenção dos alunos de modo espontâneo, e esse fator é de tamanha importância para a construção de novos saberes, pois baseado em [Machado 2015] o aluno precisa ser desafiado para que possa aprender efetivamente, conforme o conceito.

As evidências de um trabalho desenvolvido com habilidades associadas ao discente geram resultados eficazes, mesmo não havendo um método que seja global para alfabetizar, o educador é o profissional responsável por adaptar suas experiências de trabalho e aprendizagem, logo também devem se adequar à tecnologia, visto que esta já se faz presente em nossa sociedade [Jesus 2014].

Conforme as Orientações Curriculares para Ciências da Natureza, Matemática e suas Tecnologias, é importante que sejam contemplados a matemática e a tecnologia como ferramentas associadas e complementares, onde uma compreende a outra e vice-versa [Brasil 1996].

As dificuldades no ensino da matemática podem estar relacionadas a vários fatores que contribuem para a má formação dos discentes nas séries iniciais, essas dificuldades podem ser provocadas por ações metodológicas usadas pelo professor.

Portanto, os resultados negativos associados às dificuldades relacionadas à aprendizagem matemática apontam os fatores que podem levar uma pessoa a ter tanta dificuldade em dominar a parte básica. As razões dependem de como é apresentado e tratado esse assunto, como é tocada a parte emotiva do estudante. A maioria deles não consegue associar os assuntos à vida real, deste modo a aprendizagem torna-se chata e cansativa, ficando o professor com o "árduo" trabalho.

Para que os métodos e as ferramentas utilizadas pelo professor produzam os conhecimentos esperados, é preciso usar metodologias alternativas como: jogos, software, material lúdico e aula expositiva. Assim, afirma [Bertomeu 2010] a produção do material assume outra dimensão com a tomada de decisão, gerando assim uma aprendizagem ao usuário, ao invés somente de repassar informação.

Neste sentido o domínio dessa ferramenta é indispensável para professor e aluno. As tecnologias deixam as aulas de matemática criativas, e os softwares são propostas curriculares interessantes para se trabalhar com conteúdos matemáticos. A partir da concretização pode-se julgar a aptidão ao meio educacional, atendendo às propostas de ensino ao sistema. Os softwares quando são bem aplicados provocam no aluno uma reação de confiança e segurança, pois eles conseguem aproximar a teoria da prática, fazendo fluir à compreensão do assunto.

Dessa maneira visa-se a aplicação de um software com formato semelhante a um jogo, tendo como finalidade o estudo das multiplicações da tabuada e interpretação gráfica dos tipos coluna, linha e radar, objetivando unificar divertimento e conhecimentos nos assuntos 
VII Congresso Brasileiro de Informática na Educação (CBIE 2018)

Anais dos Workshops do VII Congresso Brasileiro de Informática na Educação (WCBIE 2018)

descritos, assim como verificar a potencialidade didática do software no que diz respeito à plotagem gráfica dos resultados.

O restante do trabalho é organizado da seguinte maneira: a Seção 2 apresenta os trabalhos relacionados; já a Seção 3 descreve o aplicativo Multigraf e suas funcionalidades; a metodologia empregada pode ser vista na Seção 4; por fim, a seção 5 expõe os resultados do trabalho.

\section{Trabalhos Relacionados}

Como forma de identificar os trabalhos relacionados, foram realizadas buscas na literatura e em lojas de aplicativos visando encontrar trabalhos que apresentassem aplicações e ou aplicativos direcionados ao ensino de gráficos. A Tabela 1 apresenta um comparativo envolvendo os aspectos presentes neste trabalho em relação aos demais.

Tabela 1. Trabalhos Relacionados.

\begin{tabular}{|l|l|l|l|}
\hline & Multiplicação & Gráficos & Aplicação Móvel \\
\hline [Cardoso, Giraldello and Nalva 2013] & X & - & - \\
\hline [Ferrari et al. 2014] & - & X & - \\
\hline [Rolino, Afini and Vieira 2015] & X & - & - \\
\hline [Batista et al. 2017] & X & - & - \\
\hline Este trabalho & X & X & X \\
\hline
\end{tabular}

Fonte: Elaborado pelos autores, 2018.

\section{Aplicativo Multigraf}

Nesta seção são apresentadas às funções e telas do software em estudo, com todas as opções disponíveis em cada tela, bem como conhecer as cores dos números e letras em que são expostos, os tipos de gráficos gerados por ele, e os alertas de erros e acertos. Deste modo, o aprendiz conhecerá todas as ferramentas expostas no aplicativo.

\subsection{A função que define a pontuação}

A função usada para determinar a pontuação do aluno no software, está geometricamente representada na Figura 1. Percebe-se, observando a representação, que no intervalo de $0 \mathrm{~s}$ a $1 \mathrm{~s}$ o rendimento do discente permanece constante, portanto, a lei de formação que define a função nesse intervalo será dada por $f(x)=100 \mathrm{com} 0 \leq x \leq 1$, sendo assim, a média máxima do aluno nesse intervalo será equivalente a 100. Também pela representação gráfica, é possível visualizar um declínio no rendimento do jogador no intervalo de $1 \mathrm{~s}$ a 20 s, sendo perceptível que existe uma proporção do eixo das abscissas com o eixo das ordenadas, uma vez que o rendimento do aluno é inversamente proporcional ao tempo, ou seja, quanto maior o tempo gasto para o jogador responder as perguntas, menor o valor assumido (nota) na variável y, tendo como tempo inicial, um segundo, uma vez que um intervalo menor que este não seria favorável para que alguém conseguisse responder aos questionamentos. Desta forma, é possível descrever a lei de formação que define a função no intervalo $1 \mathrm{~s}$ a $20 \mathrm{~s}$, através da equação da reta, observando os pontos no gráfico, representados nas formas de equação (I) $y-y o=m(x-x 0)$ e equação (II), $\mathrm{m}=\frac{(y-y 0)}{(x-x 0)}$, onde o valor $\mathrm{m}$ é o coeficiente angular. Por definição temos que xo e yo são o menor valor assumido e consequentemente a sua imagem, mas como trata-se de uma função inversa, seus valores são respectivamente 1 a 100 , e desta forma temos que $x=20, y=0, x 0=1, y 0=100$. Com as 
VII Congresso Brasileiro de Informática na Educação (CBIE 2018)

Anais dos Workshops do VII Congresso Brasileiro de Informática na Educação (WCBIE 2018)

variáveis definidas, os valores serão substituídos na segunda equação, resultando em $\mathrm{m}=$ $\frac{0-100}{20-1}$. Em seguida, substituindo o valor encontrado na primeira equação, observa-se que $y-100=-100 / 19(x-1)$. Desenvolvendo os cálculos obtém-se $\mathrm{y}=\frac{-100 x+100}{19}+100, \operatorname{logo}$ para $1<\mathrm{x} \leq 20$ a função é dada por $f(x)=-100 x / 19+2000 / 19$, resultando assim na lei de formação que pontua o jogo a partir de um segundo, desenvolvida pelo software.

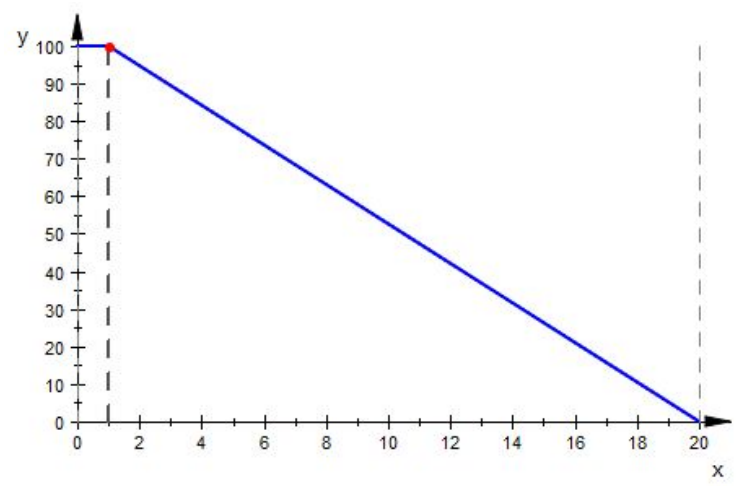

Figura 1. Representação geométrica da equação do software. Fonte: Elaborado pelos autores.

\subsection{Telas do aplicativo}

No primeiro momento, o dispositivo é reconhecido em meio aos outros no celular, por um número sete (7) e escrito abaixo "AppMultigraf” com o símbolo de multiplicação ao lado, representado pelo (x). Ao abrir o aplicativo, será disponibilizado o seguinte menu exposto abaixo, onde há uma mensagem na primeira linha com os dizeres "escolha uma opção", com as alternativas conforme a Figura 2.

Na segunda linha onde está escrito "aprender" neste opção ao ser aberto será exposto toda a tabuada de multiplicar, em cada tela uma opção de tabuada, em qualquer momento pode ser retornado ou avançado para qualquer multiplicação desejada com os respectivos botões expostos $<<>$. As cores dos números são exibidas em vermelho. A visualização é compatível com todos os celulares, pois existe a rolagem de tela, permitindo que aparelhos grandes e pequenos consiga figurar todos os números.

Esta opção serve como uma tabuada manual, só que bem mais prática, permitindo ao aluno estudar e tirar suas dúvidas a respeito de multiplicação em seu celular em qualquer lugar, já que ela está em seu próprio celular. A Figura 3 abaixo representa a janela da tabuada

Na opção "jogar" o jogador tem que estar habituado com todas as multiplicações. Ao clicar nesta opção, abrirá uma nova janela. Nela serão geradas perguntas sobre multiplicações abrangendo toda a tabuada, sendo estas apresentadas aleatoriamente. Será disponibilizado o teclado com os números de zero a nove, ficando a cargo da habilidade do jogador em forma o número correto da resposta. As perguntas não apresentam alternativas, estimulando ainda mais o aprendizado assim também despertar o espírito competitivo como em qualquer outro jogo virtual, diversificando a matemática. Ao final de todas as perguntas feitas é lançado uma mensagem ao usuário de "parabéns" indicando que foram respondidas todas as perguntas, mesmo que em alguns casos incorretas, quando não completado esse ciclo por algum motivo, e desiste de continuar naquele momento, não aparece a mensagem. 
VII Congresso Brasileiro de Informática na Educação (CBIE 2018)

Anais dos Workshops do VII Congresso Brasileiro de Informática na Educação (WCBIE 2018)

\begin{tabular}{|l|}
\hline Escolha uma Opção: \\
Aprender \\
Jogar \\
Desempenho \\
Sobre \\
\end{tabular}

Figura 2. Tela inicial.

Fonte: Multigraf, 2018.

\begin{tabular}{|l}
\hline$<$ \\
$2 \times 1=2$ \\
$2 \times 2=4$ \\
$2 \times 3=6$ \\
$2 \times 4=8$ \\
$2 \times 5=10$ \\
$2 \times 6=12$ \\
$2 \times 7=14$ \\
$2 \times 8=16$ \\
$2 \times a=18$
\end{tabular}

Figura 3. Tela aprender.

Fonte: Multigraf, 2018.

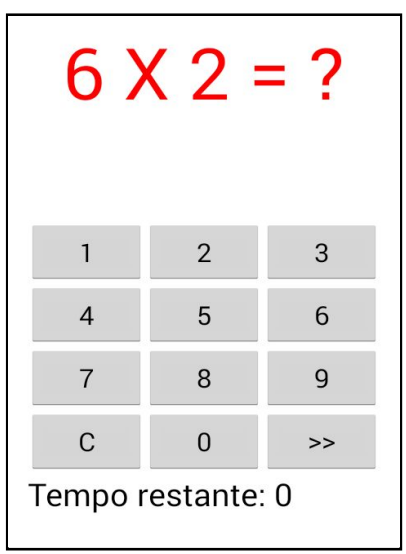

Figura 4. Tela jogar.

Fonte: Multigraf, 2018.

Quando uma pergunta é respondida incorretamente ou é excedido o tempo limite de vinte segundos, é disparado um alerta que inclui a vibração do celular, notificando o competidor. Na Figura 4 está representada essa tela.

Na opção "desempenho", é possível observar o desenvolvimento de todo o jogo, permitindo ao jogador, analisar onde ele está indo bem ou mal nas operações de multiplicação, permitindo ao jogador identificar onde ele precisa melhorar. Deste modo, o competidor se sente estimulado a estudar a tabuada cada vez mais, para assim tentar obter uma atuação maior no jogo e poder superar seu próprio recorde ou de outros colegas.

O resultado de cada partida é analisado graficamente e isso é outro ponto forte deste software, pois engrandece o conhecimento do participante, permitindo assim observar os erros e acertos de uma maneira bem diferente, se diferenciando de outros aplicativos semelhante que não apresentam essa análise, mas sim apenas um número representando o resultado. São disponibilizados neste software três tipos de gráficos para a interpretação dos resultados, sendo eles o de coluna, linha e radar, ficando a disposição do jogador interpretá-los, pois são expostos três pequenos botões, bastando clicar em cima deles para mudar de gráfico.

O primeiro deles é bastante conhecido e fácil de ser interpretado. Para cada multiplicação é gerado uma coluna onde pode assumir um valor no eixo y, entre 0 e 100 . Desta forma é gerado um gráfico para cada tabuada, sendo ao todo oito gráficos. As colunas são ilustradas em uma cor violeta, podendo o competidor destacar uma barra do gráfico ao clicar em cima dela, e esta muda a sua cor para o roxo, se destacando. A Figura 5 ilustra essa tela.

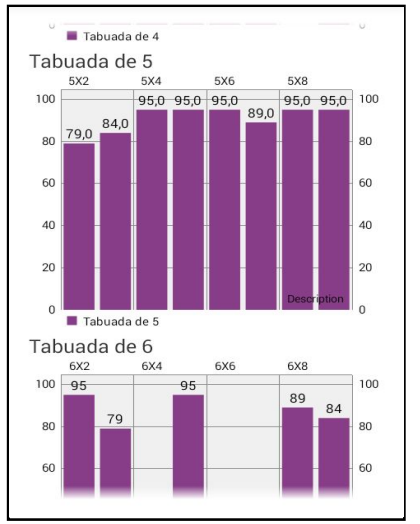

Figura 5: Gráfico de Colunas Fonte: Multigraf, 2018.

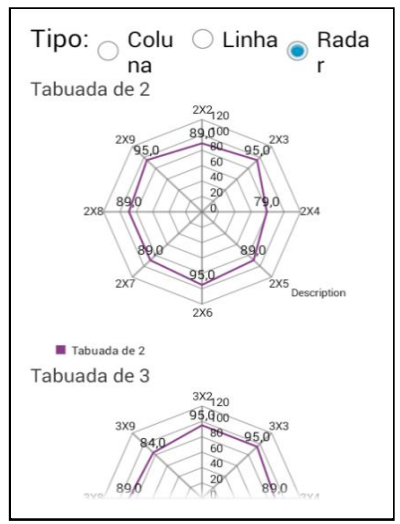

Figura 6: Gráfico Radar. Fonte: Multigraf, 2018.

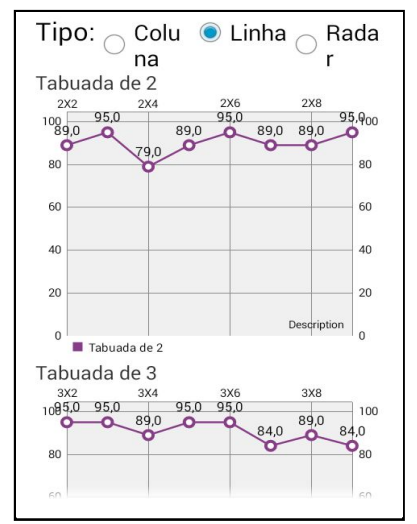

Figura 7: Gráfico de Linhas. Fonte: Multigraf, 2018. 
O gráfico radar também é utilizado no aplicativo, sendo que ele já possui uma interpretação mais apurada, com uma boa qualidade de associação de dados. Todos eles apresentam os resultados entre 0 e 100. Esta tela está representada na Figura 6.

O outro gráfico trabalhado é o de linhas, e mesmo não sendo tão utilizado como o colunas, é importante saber analisar dados por ele representados. Nele é possível observar o percurso em todos os momentos, onde se teve um declive ou um crescimento de acertos como mostra a Figura 7.

Os gráficos são salvos a cada jogada finalizada, e ficam disponível para consultas a qualquer momento. Ao iniciar uma nova partida, estes dados são armazenados na memória interna do aparelho, e o gráfico anterior é substituído pelos resultados da próxima jogada. Desta forma os resultados anteriores poderão ser consultados nos arquivos do aparelho, já que estão devidamente armazenados.

\section{Metodologia}

Souza [2006] apresentam duas formas de avaliação de software educativo: a formativa e a objetiva. A maneira consiste em um meio para acompanhar a utilização de um software educativo dentro de um ambiente de aprendizagem real onde a primeira, os alunos compartilham com o objeto de conhecimento, e na segunda presume o uso de lista de critérios, por distintos autores.

Para a realização desta pesquisa foram envolvidos vinte alunos de uma turma que continha trinta e dois alunos. Os alunos cursam o nono ano na Escola Professora Lourdes Costa localizado no município de Icó-Ceará. Eles aceitaram participar do estudo de forma voluntária, de modo a contribuir para a melhoria na educação como todo. Os demais alunos não puderam participar por não estarem presentes na primeira ou na segunda coleta de dados.

Durante as práticas desenvolvidas em sala utilizando o software e aplicação do questionário que envolvia quatro perguntas, os alunos executaram suas atividades de forma individual. Os discentes que participaram da atividade proposta pela pesquisa, estavam inicialmente com o software devidamente instalado em seus aparelhos celulares e houve alguns testes iniciais, para que os mesmos tomassem conhecimento dos recursos do aplicativo. Em seguida, foram entregues aos alunos os questionários para que fossem devidamente respondidos de forma individual, conforme o fluxo da atividade. Ao final da aplicação, foram coletadas as informações de cada aluno, por meio da transferência desses dados para um notebook.

Os dados foram estudados estatisticamente, objetivando uma ampla compreensão dos resultados obtidos, os quais serão discriminado o desempenho dos discentes nos assuntos de multiplicação e interpretação gráfica, como reflexo da aprendizagem.

\section{Resultados}

\subsection{Primeiro encontro}

Para facilitar a manipulação dos dados, os participantes da pesquisa foram identificados da seguinte forma: aluno um como A1, aluno dois como A2 e assim sucessivamente até o A20. $\mathrm{Na}$ primeira amostra, foi pontuada a média de acertos de cada aluno executada no software, conforme mostrado na Tabela 2. 
VII Congresso Brasileiro de Informática na Educação (CBIE 2018)

Anais dos Workshops do VII Congresso Brasileiro de Informática na Educação (WCBIE 2018)

Tabela 2 - Médias dos alunos mediante execução no software no primeiro encontro.

\begin{tabular}{|c|c|c|}
\hline $\begin{array}{l}\mathrm{A} 1=66.5, \\
\mathrm{~A} 4=82, \\
\mathrm{~A} 7=57.2, \\
\mathrm{~A} 10=58.6, \\
\mathrm{~A} 13=84.8, \\
\mathrm{~A} 16=60.7, \\
\mathrm{~A} 19=56,3,\end{array}$ & $\begin{array}{l}\mathrm{A} 2=58.9, \\
\mathrm{~A} 5=51.7, \\
\mathrm{~A} 8=75.2, \\
\mathrm{~A} 11=89, \\
\mathrm{~A} 14=66.5, \\
\mathrm{~A} 17=77, \\
\mathrm{~A} 20=69,5\end{array}$ & $\begin{aligned} \mathrm{A} 3 & =70.1, \\
\mathrm{~A} 6 & =68.9, \\
\mathrm{~A} 9 & =71.2, \\
\mathrm{~A} 12 & =93.6, \\
\mathrm{~A} 15 & =75.2, \\
\mathrm{~A} 18 & =44.6,\end{aligned}$ \\
\hline
\end{tabular}

Fonte: Elaborado pelos autores.

Neste primeiro momento, quando aplicado o questionário com as quatro perguntas e a execução do software, pôde-se observar que houve um aluno com a média inferior a cinquenta por cento, e seis abaixo de sessenta por cento, demonstrando a fragilidade de conhecimento deles em multiplicações. Nesta primeira amostra, obteve-se uma média geral de 68,8 . Analisando de forma mais aprofundada, tem-se que: a população estatística é formada por um grupo de 20 alunos, que a unidade estatística é representada por cada aluno desta pesquisa e como variável estatística têm-se as médias de cada aluno, conforme execução no software.

Houve uma distribuição de dados agrupados, onde foi possível tomar conhecimento da frequência relativa acumulada. Os valores são dados em porcentagem, visto que deste modo se torna mais claro sua compreensão e análise [Giovanni, Bonjorno e Junior 2002]. A menor média foi 44,6, e a maior foi 93,6, podendo-se agrupá-las em intervalos de amplitude 10, ou seja, [40; 50[, [50;60[, [60; 70[, [70; 80[, [80; 90[ e [90; 100[. Na Tabela 3 estão os dados dessa frequência;

Tabela 3 - Distribuição de frequências das médias do primeiro encontro conforme execução no software

\begin{tabular}{|c|c|c|c|c|}
\hline $\begin{array}{c}\text { Classes ou } \\
\text { intervalos }\end{array}$ & Frequência & $\begin{array}{c}\text { F. Absoluta } \\
\text { acumulada }\end{array}$ & F. relativa & $\begin{array}{c}\text { F.relativa } \\
\text { acumulada }\end{array}$ \\
\hline$[40 ; 50[$ & 1 & 1 & $1 / 20=5 \%$ & $1 / 20=5 \%$ \\
\hline$[50 ; 60[$ & 5 & $1+5=6$ & $5 / 20=25 \%$ & $6 / 20=30 \%$ \\
\hline$[60 ; 70[$ & 5 & $6+5=11$ & $5 / 20=25 \%$ & $11 / 20=55 \%$ \\
\hline$[70 ; 80[$ & 5 & $11+5=16$ & $5 / 20=25 \%$ & $16 / 20=80 \%$ \\
\hline$[80 ; 90[$ & 3 & $16+3=19$ & $3 / 20=15 \%$ & $19 / 20=95 \%$ \\
\hline$[90 ; 100[$ & 1 & $19+1=20$ & $1 / 20=5 \%$ & $20 / 20=100 \%$ \\
\hline
\end{tabular}

A Tabela 3 de distribuição de frequência demonstra em detalhes os resultados obtidos nessa análise. Conhecendo o grau de dificuldade que o software apresenta, pôde-se classificar os alunos em péssimos, quando suas médias foram abaixo de sessenta, bons quando obtiveram médias entre sessenta e oitenta, e excelente, quando alcançaram médias no intervalo de oitenta a cem. Abaixo, seguem as informações em detalhes: (i) $25 \%$ dos alunos se concentraram em cada intervalo [50;60[, [60;70[, [70;80[, ou seja, onde houve as maiores concentrações, onde tem-se discentes péssimos e bons; (ii) $30 \%$ são considerados péssimos, com médias menores que 60; (iii) 50\% tiveram a média entre 60 e 80 , sendo considerados bons; (iv) $15 \%$ tiveram a média maior que 80 , se enquadrando como excelentes; ( $v$ ) $5 \%$ dos alunos tiveram média acima de 90 , alcançando a maior média. 
VII Congresso Brasileiro de Informática na Educação (CBIE 2018)

Anais dos Workshops do VII Congresso Brasileiro de Informática na Educação (WCBIE 2018)

Pode-se perceber que a metade dos alunos apresentou média satisfatória, ficando entre sessenta e oitenta. Poucos alunos atingiram uma média acima de noventa, sendo que, nenhum aluno obteve nota máxima. Assim, é possível constatar um resultado válido por parte dos alunos no primeiro encontro, induzindo à uma contribuição válida no uso do Multigraf no que tange a aprendizagem da multiplicação.

\subsection{Segundo encontro}

No segundo encontro foram analisados estatisticamente os resultados executados no software, da mesma forma como haviam sido classificados anteriormente, gerando as médias apresentadas no Quadro 3.

Quadro 3 - Médias dos alunos mediante execução no software no segundo encontro $\mathrm{A} 1=86.5, \mathrm{~A} 2=72.9, \mathrm{~A} 3=90,2, \mathrm{~A} 4=84.8, \mathrm{~A} 5=70,8 \mathrm{~A} 6=75.7, \mathrm{~A} 7=73.2, \mathrm{~A} 8=85, \mathrm{~A} 9=67.2$, $\mathrm{A} 10=73.2, \mathrm{~A} 11=79, \mathrm{~A} 12=95, \mathrm{~A} 13=92, \mathrm{~A} 14=85, \mathrm{~A} 15=76,1, \mathrm{~A} 16=87.7, \mathrm{~A} 17=82$, $\mathrm{A} 18=61.6, \mathrm{~A} 19=82, \mathrm{~A} 20=80$.

Fonte: Elaborado pelos autores.

Analisando essas médias, percebe-se que não houve nenhuma nota inferior a cinquenta por cento, indicando resultados expressivos como média geral igual a 80 . Isso significa uma progressão significativa de $16.2 \%$ em relação à primeira amostra. Como na primeira amostra, os dados foram agrupados em um quadro, onde se tem como média mínima o valor de 61.6 e a máxima o valor de 95 . Estes foram agrupados em intervalos de amplitude 10, ou seja, [60;70[, [70;80[, [80;90[ e [90; 100[. Com base nesses dados, foi construído Quadro 4 com a distribuição de frequência, para melhor entendimento dos resultados verificados.

Quadro 4 - Distribuição de frequências das médias do segundo encontro conforme execução no software

\begin{tabular}{|c|c|c|c|c|}
\hline $\begin{array}{c}\text { Classes ou } \\
\text { intervalos }\end{array}$ & Frequência & $\begin{array}{c}\text { F.Absoluta } \\
\text { acumulada }\end{array}$ & F. relativa & $\begin{array}{c}\text { F.relativa } \\
\text { acumulada }\end{array}$ \\
\hline$[60 ; 70[$ & 2 & 2 & $2 / 20=10 \%$ & $2 / 20=25 \%$ \\
\hline$[70 ; 80[$ & 7 & $2+7=9$ & $7 / 20=35 \%$ & $9 / 20=45 \%$ \\
\hline$[80 ; 90[$ & 8 & $9+8=17$ & $8 / 20=40 \%$ & $17 / 20=85 \%$ \\
\hline$[90 ; 100[$ & 3 & $17+3=20$ & $3 / 20=15 \%$ & $20 / 20=100 \%$ \\
\hline
\end{tabular}

Fonte: Elaborado pelos autores.

Os dados calculados foram comparados com os mesmos resultados obtidos no primeiro encontro, buscando confrontar os resultados discriminados e verificando o comportamento dessas informações. Abaixo segue o detalhamento do que aconteceu de diferente nesses dois momentos: (I) 40\% dos alunos tiveram a média entre 80 e 90 , ou seja, a maior concentração considerada excelente; (II) Não foram pontuadas médias abaixo de 60, ou seja, não tiveram alunos classificados como péssimos; (III) $100 \%$ tiveram a média maior que 60 , sendo considerados bons; (IV) $40 \%$ pontuaram com média acima de 80 , tido como excelentes; (V) 15\% tiveram as médias maiores que 90, ou seja, obtiveram as maiores médias.

\subsection{Análise Gráfica}

Com todos os dados expostos, foram feitas algumas observações e comparados os dados do primeiro encontro com o segundo, tanto os resultados trabalhado no software como também o questionário de cada aluno, onde podemos chegar a considerações válidas sobre este estudo 
VII Congresso Brasileiro de Informática na Educação (CBIE 2018)

Anais dos Workshops do VII Congresso Brasileiro de Informática na Educação (WCBIE 2018)

utilizando esta ferramenta tecnológica como intermediador de conhecimentos para facilitar a aprendizagem matemática, nas multiplicações e leitura de gráficos.

Em relação à utilização do software, observa-se que, no primeiro encontro, houve médias inferiores a cinquenta, e as maiores concentrações de médias, ficaram entre cinquenta e oitenta. Acima de noventa, houve uma distribuição razoável. Observando as médias do segundo encontro, nota-se que houve um destaque onde a maioria dos alunos teve as médias entre oitenta e noventa, demonstrando relevância de conhecimentos na tabuada de multiplicação. As médias acima de noventa apresentaram um sutil crescimento. Também é visível que as menores notas ficaram acima de cinquenta, embora nenhum aluno tenha conseguido atingir cem por cento na pontuação, ou seja, não responderam todas as multiplicações corretas em menos de um segundo. Já era previsível que objetivassem algumas perguntas nesse intervalo, mas não todas.

$\mathrm{Na}$ segunda pergunta, fica visível que teve um aumento de alunos com a opção "excelente" em interpretar os três tipos de gráficos em estudo, e que no segundo encontro foi verificado que os discentes não assinalaram a opção "péssimo". Desta forma, verificam-se resultados positivos neste item. Analisando a terceira pergunta, o destaque vai para o gráfico de barras, onde metade dos alunos responderam entendê-lo e 5\% afirmam entender os três tipos. Já no segundo encontro, 50\% responderam compreender dois tipos, colunas e linhas, e $35 \%$ declaram entender os três tipos. Neste caso fica evidente que há uma abrangência de conhecimentos acerca de gráficos muito favoráveis.

Veja que, na quarta pergunta que tinha como objetivo identificar quais números os alunos apresentavam maiores dificuldades, foram pontuados os números de cinco a nove, e que nos dois encontros permaneceram quase que inalterados, ou seja, mesmo com melhorias com a utilização do software estes números são os que mais incomodam aos alunos. Diante destas informações, pode-se pensar de modo positivo, a utilização deste software como intermediador da aprendizagem, pois o mesmo apresentou eficiência, pois os testes em que envolveram a sua metodologia apresentaram resultados satisfatórios.

O saldo final desta observação fica em aberto, mas com exemplo positivo de que é possível trabalhar a matemática de uma forma mais dinâmica e menos formal, com auxílio de ferramentas tecnológicas relacionadas à áreas específicas.

\section{Considerações Finais}

O presente trabalho mostrou que, com os novos utensílios tecnológicos, é possível melhorar o rendimento dos alunos na obtenção de conhecimentos matemáticos dentro da sala de aula. As Tecnologias da Informação e Comunicação são apontadas como uma ponte para uma compreensão mais dinâmica dos conteúdos desde a sua criação, onde seria capaz de interligar conhecimentos imaturos a outros que necessitasse de uma abstração mais apurada.

A pesquisa realizada aponta que a aceitação deste trabalho pelos alunos foi bastante empolgante, contando com a contribuição de todos em tudo o que foi solicitado. Os alunos passaram por dois momentos importantes, os quais foi possível identificar o nível de conhecimento dos mesmos em relação aos três gráficos: coluna, linhas e radar, como também sobre a tabuada de multiplicação.

Era visível a dificuldade dos alunos quando tiveram o primeiro contato com o software, tanto que os resultados obtidos no primeiro momento foram bem diferentes quando comparados ao do segundo, demonstrando assim um crescimento de domínio em relação aos dois pontos abordados no estudo. Isso demonstra a eficiência das TICs, mesmo que o software tenha abordado o campo de estudo de forma limitada. Deste modo, houve resultados 
VII Congresso Brasileiro de Informática na Educação (CBIE 2018)

Anais dos Workshops do VII Congresso Brasileiro de Informática na Educação (WCBIE 2018)

animadores que encorajam o uso dessas novas ferramentas como uma proposta para os profissionais docentes.

Os objetivos traçados nesta análise foram alcançados com êxito, pois envolveram alunos e professores, que contribuíram de forma espontânea, tornando o resultado aceitável. Diante disso, o recurso em estudo mostra eficácia na maneira de como foi representado estes termos matemáticos. Deste modo, este estudo pode influenciar outros trabalhos que podem ter como base, os resultados gerados neste.

Como trabalhos futuros, sugere-se a inserção da geometria plana, com ênfase na abordagem e na identificação de figuras planas pelos nomes, assim como nos ângulos conhecidos e os valores dos ângulos internos de cada figura.

\section{Referências}

Batista, E. J. S., Mioto, J., Bogarim, C. A. C., Lima, A., \& Araujo, Q. (2017, October). As Aventuras de Calculino: jogo para ensino de raciocínio lógico. In Anais dos Workshops dr Congresso Brasileiro de Informática na Educação (Vol. 6, No. 1, p. 451).

Bertomeu, J. V. C. (2010) “Criação Visual e Multimídia”, Cengage Learning, 2010.

Brasil. (1996). "Lei no 9394, de 20 de dezembro de 1996". Retrieved January 18, 2018, from http://www.planalto.gov.br/ccivil_03/Leis/L9394.htm

Cardoso, A., Giraldello, A. G., \& Nalva Ap M, B. (2013). Tabuada Legal: um jogo sério para o ensino de multiplicações. In Brazilian Symposium on Computers in Education (Simpósio Brasileiro de Informática na Educação-SBIE) (Vol. 24, No. 1, p. 376).

Ferrari, S. C., Prado, R. C., Paulo, F. A., \& Alexandre, R. M. (2014). SGESTAT-Software para cálculos e gráficos estatísticos: uma ferramenta computacional para o apoio do ensino-aprendizagem de Estatística. In Anais dos Workshops do Congresso Brasileiro de Informática na Educação (Vol. 3, No. 1, p. 135).

Giovanni, J. R., Bonjorno, J. R. \& Junior, J. R. G. (2002) “Matemática completa”, Bela VistaSão Paulo: Ftd.

Jesus, G. (2014). “Jogos Educativos”. Retrieved January 17, 2018, from http://www.artigonal.com/jogos-artigos/jogos-educativos.

Machado, I. G. (2015). "Educação e tecnologia: a influência desta aliança na educação de crianças e adolescentes". Retrieved January 17, 2018, from http://www.artigonal.com/

Pantaleão, E., Amaral, L. R., \& Braga, G. (2017). An approach based on Robocode environment for teaching programming in high school. In: Brazilian Journal of Computers in Education, 25(03), 95. doi:/10.5753/rbie.2017.25.03.95.

Rolino, J., Afini, D., \& Vieira, G. (2015, October). Pirâmide Multiplicativa: um jogo sério para a memorização da tabuada. In Brazilian Symposium on Computers in Education (Simpósio Brasileiro de Informática na Educação-SBIE) (Vol. 26, No. 1, p. 617).

Souza, M. F. C. (2006). Um ambiente de apoio à seleção de software educativo, $80 \mathrm{f}$. Mestrado e Doutorado em Ciência da Computação, Universidade Federal do Ceará. 